\title{
Survival score to characterize prognosis in inoperable stage III NSCLC after chemoradiotherapy
}

\author{
Julian Taugner ${ }^{1}$, Lukas Käsmann ${ }^{1,2,3}$, Chukwuka Eze ${ }^{1,2}$, Maurice Dantes ${ }^{1,2}$, Olarn Roengvoraphoj ${ }^{1}$, \\ Kathrin Gennen ${ }^{1}$, Monika Karin ${ }^{1}$, Oleg Petruknov ${ }^{1}$, Amanda Tufman ${ }^{2,4}$, Claus Belka ${ }^{1}$, Farkhad Manapov ${ }^{1,2}$ \\ ${ }^{1}$ Department of Radiation Oncology, University Hospital Munich (LMU), München, Germany; ${ }^{2}$ Comprehensive Pneumology Center Munich \\ (CPC-M), Member of the German Center for Lung Research (DZL), Munich, Germany; ${ }^{3}$ German Cancer Consortium (DKTK), partner site \\ Munich, Munich, Germany; ${ }^{4}$ Division of Respiratory Medicine and Thoracic Oncology, Department of Internal Medicine V, Thoracic Oncology \\ Centre Munich, Ludwig-Maximilians University, München, Germany \\ Contributions: (I) Conception and design: All authors; (II) Administrative support: All authors; (III) Provision of study materials or patients: All \\ authors; (IV) Collection and assembly of data: J Taugner, L Käsmann, C Eze, O Roengvoraphoj, K Gennen, M Karin, O Petruknov, F Manapov; \\ (V) Data analysis and interpretation: J Taugner, L Käsmann, C Eze, O Roengvoraphoj, F Manapov; (VI) Manuscript writing: All authors; (VII) Final \\ approval of manuscript: All authors. \\ Correspondence to: Julian Taugner. Guardinistr, 148, 81375 Munic, Germany. Email: Julian.Taugner@med.uni-muenchen.de.
}

\begin{abstract}
Background: Stage III non-small cell lung cancer (NSCLC) represents a heterogeneous disease regarding principal patient- and tumor characteristics. A simple score may aid in personalizing multimodal therapy.

Methods: The data of 99 consecutive patients with performance status ECOG 0-1 treated until the end of 2016 with multimodal approach for inoperable NSCLC (UICC $7^{\text {th }}$ edition stage IIIA/B) were evaluated. Patient- and tumor-related factors were examined for their impact on overall survival. Factors showing a negative association with prognosis were then included in the score. Three subgroups with low, intermediate and high-risk score were defined. The results were then validated in the prospective cohort, which includes 45 patients.

Results: Most Patients were treated with concurrent (78\%) or sequential (11\%) chemoradiotherapy. 53\% received induction chemotherapy. Median survival for the entire cohort was 20.8 (range: 15.3-26.3) months. Age $(\mathrm{P}=0.020)$, gender $(\mathrm{P}=0.007)$, pack years $(\mathrm{P}=0.015)$, tumor-associated atelectasis $(\mathrm{P}=0.004)$ and histology $(\mathrm{P}=0.004)$ had a significant impact on overall survival and were scored with one point each. Twelve, 59 and 28 patients were defined to have a low (0-1 points), intermediate ( $2-3$ points) and high-risk ( $4-5$ points) score. Median survival, 1-, 2- and 3-year survival rates were not reached, 100\%, 83\% and $67 \%$ in the low, 22.9 months, $80 \%, 47 \%$ and $24 \%$ intermediate and 13.7 months, $57 \%, 25 \%$ and $18 \%$ high-risk patients, respectively $(\mathrm{P}<0.001)$. Median survival was not reached in prospective cohort; analysis has revealed a trend for the 1 -year survival rates with $100 \%$ for the low, $93 \%$ intermediate and $69 \%$ high-risk patients $(\mathrm{P}=0.100)$. Conclusions: The score demonstrated remarkable survival differences in inoperable stage III NSCLC patients with good performance status receiving multimodal therapy.
\end{abstract}

Keywords: Non-small cell lung cancer (NSCLC); survival-score; prognostic factors; multimodal therapy

Submitted Jul 07, 2019. Accepted for publication Sep 05, 2019.

doi: $10.21037 /$ tlcr.2019.09.19

View this article at: http://dx.doi.org/10.21037/tlcr.2019.09.19

\section{Introduction}

Lung cancer is the leading cause of cancer-related mortality worldwide, accounting for $19.4 \%$ of all cancer-related deaths $(1,2)$. In 2012, lung cancer resulted in 1.6 million deaths worldwide (3), making it the leading cause of cancer- related death for men and second leading for women worldwide and in Germany with a rising tendency (4).

Up to date, treatment algorithm for patients with nonsmall cell lung cancer (NSCLC) depend on patients' general condition and tumor stage. Patients with locally-advanced 
NSCLC are treated with cost-intensive multimodal therapy consisting of chemotherapy, thoracic irradiation, surgery and checkpoint inhibition (5-12). Stage III NSCLC is a very heterogeneous disease regarding principal patient characteristics such as age, gender, comorbidities and tumor features like histology, localization, tumor dimensions and specificity of lymph node involvement. Furthermore, a multimodal approach can be burdensome and may lead to deterioration of patients' general condition, because of potential hematological and non-hematological toxicity $(13,14)$. Historically, survival of inoperable stage III NSCLC after multimodal approach shows a significant variety $(15,16)$. All of these factors considered, tailored multimodal therapy should be considered the next step for locally-advanced NSCLC.

Physician decision-making may be facilitated by using different prognostic scores (17). Alexander et al. recently proposed the "Lung Cancer Prognostic Index" (LCPI), considering different tumor stages and including established and novel factors for the prediction of survival after NSCLC diagnosis (18). The LCPI was defined by 9 variables with different point allocations resulting in a total of 28 points that ultimately classifies the patients into four risk groups. For incurable patients, a more simplistic approach is defined by the Montreal prognostic score depending on clinical biomarkers (19) or the score proposed by Rades et al. for patients receiving palliative irradiation (20).

Our study, however, was dedicated to scale heterogeneity in inoperable stage III NSCLC patients with good PS ECOG 0-1 treated with chemoradiotherapy in curative intent and develop a simple heterogeneity score which can be easily adopted in clinical routine.

\section{Methods}

\section{Patients}

Data were collected on a total of 99 consecutive patients treated with curative-intent multimodal treatment for UICC 7th edition stage IIIA/B NSCLC. All patients gave their informed consent to treatment and the use of acquired data for research purposes. There was ethical committee approval to analyze and publish the patients' data.

All patients were treated at a single tertiary cancer center. Pre-treatment evaluation included: patients' history i.e. tobacco consumption, ECOG PS and comorbidities, pulmonary function testing, radiographic imaging including computed tomography (CT) for all patients and positron emission tomography (PET)-CT in $94 \%$ of patients, routine blood work to assess kidney, liver function and blood cell count. Cranial contrast-enhanced MRI was performed in 28 patients before the start of multimodal treatment, all other patients received contrast-enhanced head CT.

Tumor histology was obtained via transbronchial biopsy in 80 patients, via CT-guided-biopsy in 9 patients and with mediastinoscopy in 10 patients. Histological or cytological confirmation of nodal involvement was performed in 67 patients.

Treatment was then discussed and planned in multidisciplinary tumor boards, where all patients tumors were initially classified as inoperable by experienced thoracic surgeons. Therapeutic approach was than discussed with each individual patient.

Patients with initial performance status ECOG $>1$, recurrent disease or with another neoplasia at initial diagnosis were excluded. Also, we excluded patients who underwent surgery before irradiation as well as those who received SABR or hypofractionated radiotherapy and those treated with primary palliative intent. All patients had follow-up data available until July 2018.

Validation of the score was performed in our own prospective patient cohort with same characteristics as above-mentioned retrospective cohort. To be comparable, the cohort consists of 45 consecutive Patients treated from 01.01.2017 until the implementation of adjuvant durvalumab therapy in our institution.

\section{Multimodal approach}

Treatment was planned and delivered at a single tertiary cancer center. Radiation planning and delivery were done while patients were supine, with their arms positioned overhead in WingSTEP ${ }^{\mathrm{TM}}$ (Innovative Technologie Völp, Innsbruck, Austria), based on PET-CT in treatment position and conventional Planning-CT-scans. The gross tumor volume (GTV) was defined as the primary tumor and any regionally involved nodes either positive on pretreatment PET-CT or $>1 \mathrm{~cm}$ short axis on conventional CT. In patients receiving induction chemotherapy, only residual tumor volume was contoured. A margin of $5-6 \mathrm{~mm}$ in all dimensions was added to the GTV to generate the clinical target volume (CTV) taking into consideration anatomical borders and organs at risk. The planning target volume (PTV) margin was $6-8 \mathrm{~mm}$ 
beyond the CTV. Three-dimensional (3D) conformal radiotherapy was delivered to the primary tumor and involved lymph nodes to a median total dose of $66 \mathrm{~Gy}$. Elective nodal irradiation (ENI) included directly adjacent nodal stations and was delivered to a total dose of 45-54 Gy in $85 \%$ of patients. Radiotherapy was delivered on a Linear accelerator (LINAC) with megavoltage capability (6-15 MV) using 3D-CRT in $60 \%$ of patients and Intensitymodulated radiotherapy (IMRT) in $40 \%$ of patients. Imageguidance was performed with cone-beam CT two or three times a week. All plans were reviewed by several radiation oncologists for quality assurance and appropriateness of treatment.

\section{Patient follow-up}

Median follow up for the entire cohort achieved 17.2 months (range, 2.2-92.1 months). For the first 2 years after therapy, all patients underwent CT or PET-CT scans, routine blood works, lung function testing and clinical examination every 3 months and afterwards twice a year. Cranial contrast-enhanced MRI and bone-scintigraphy were performed if clinically indicated. Treatment-related toxicity was graded retrospectively, according to the National Cancer Institute's Common Terminology Criteria for Adverse Events, version 3.0.

Local and loco-regional progression (LP) and new distant metastases (DM) were documented with CT, PETCT and MRI scans. Histological or cytological verification of progressive disease was not obligatory. Event free survival was calculated as the time until LP, DM or death from the first day of radiation therapy. Overall survival (OS) was calculated from the date of initial diagnosis.

Treatments of recurrent and/or progressive disease were tracked in the database.

\section{Statistical analysis}

All statistics were performed with IBM SPSS version 25. Survival curves were estimated with the KaplanMeier method. A variety of potential patient- and tumorassociated prognostic factors were analyzed. Cut-offs for non-binary factors were defined by trial and error only using round numbers. Impact on OS was calculated by univariate analysis using Log-rank test. Factors showing a significant negative association with patient prognosis $(\mathrm{P}<0.05)$ were included in the score and all scored with one point each, to keep the score simple. Furthermore, multivariate analysis using Cox-regression was performed with the predictors significant on univariate analysis. Three prognostic subgroups with low ( $0-1$ points), intermediate (2-3 points) and high (4-5 points) risk score were defined and compared using the Kaplan-Meier method for survival analysis and Kruskal-Wallis-Test for patient-, tumor- and treatment characteristics.

\section{Results}

\section{Patient- and tumor characteristics}

A summary of patient and tumor characteristics is shown in Table 1.

The majority (63\%) were males, median age at diagnosis was 67.4 years (range: $43-88$ years). ECOG-PS before treatment was 0 in 48 patients and 1 in 51 patients. More patients had stage IIIB (UICC $7^{\text {th }}$ edition) disease $(56 \%)$, an absolute majority had T-stage $3(30 \%)$ or $4(40 \%)$ and $\mathrm{N}$-stage $2(36 \%)$ or 3 (44\%). Median tobacco consumption was $40 \mathrm{PY}, 39 \%$ suffered from COPD and $9 \%$ of patients were never smokers.

Squamous cell carcinoma (SCC) was diagnosed in $42 \%$ of patients, adenocarcinoma in $50 \%$ and not otherwise specified (NOS) in $8 \%$ at initial diagnosis.

\section{Multimodal treatment characteristics}

A summary of the multimodal approach characteristics is listed in Table 2 .

The absolute majority of patients were treated with conventional concurrent chemoradiotherapy (CRT) to a total dose $\geq 60$ Gy (68\%). Ten percent of patients were treated with radiotherapy alone, because of poor kidney function or rejection of chemotherapy. Eleven percent were treated in the sequential mode, because they were not feasible for concurrent after induction chemotherapy. Fifty-two percent completed at least one cycle of induction chemotherapy before CRT (standard intentional strategy until mid-2013). The predominant concurrent chemotherapy regimen consisted of cisplatin given intravenously at a dose of $20 \mathrm{mg} / \mathrm{m}^{2}$ on days $1-4$ and oral vinorelbine (navelbine) $50 \mathrm{mg} / \mathrm{m}^{2}$ on days 1,8 , and 15 , every 4 weeks for two courses ( $46 \%$ of patients). Median time to CRT start was 2.5 months (range, 0.07-12.22 months). CRT was completed as planned by $95 \%$ of the patients with a median total dose of $66 \mathrm{~Gy}$ (range, 45-70 Gy). Five patients (5\%) could not complete 
Table 1 Patient and tumor characteristics

\begin{tabular}{|c|c|c|c|c|c|}
\hline Patient characteristics & All patients & Low risk & Inter-mediate risk & High risk & $\begin{array}{l}\text { P value for OS impairment (Log- } \\
\text { Rank; Mantel-Cox) }\end{array}$ \\
\hline Points in risk score & $\mathrm{n}=99$ & $\mathrm{n}=12$ & $\mathrm{n}=59$ & $n=28$ & $<0.001$ \\
\hline Range & $0-5$ & $0-1$ & $2-3$ & $4-5$ & \\
\hline \multicolumn{6}{|l|}{ Age, years } \\
\hline Range & $43-88$ & $43-78$ & $43-88$ & $61-84$ & \\
\hline$>60$ years & $73(74 \%)$ & $3(25 \%)$ & $42(71 \%)$ & $28(100 \%)$ & 0.02 \\
\hline Gender & & & & & 0.007 \\
\hline Male & $62(63 \%)$ & $3(25 \%)$ & $31(53 \%)$ & $28(100 \%)$ & \\
\hline Range & $0-150$ & $0-40$ & $0-150$ & $20-90$ & \\
\hline$\geq 20 \mathrm{PY}$ & $81(82 \%)$ & $3(25 \%)$ & $50(85 \%)$ & 28 (100\%) & 0.015 \\
\hline Atelectasis before RT & $10(10 \%)$ & $0(0 \%)$ & $7(12 \%)$ & $3(11 \%)$ & 0.004 \\
\hline \multicolumn{6}{|l|}{ Tumor histology } \\
\hline Non-adenocarcinoma & $50(51 \%)$ & $0(0 \%)$ & $22(37 \%)$ & $28(100 \%)$ & 0.011 \\
\hline Adenocarcinoma & $49(49 \%)$ & $12(100 \%)$ & $37(63 \%)$ & $0(0 \%)$ & \\
\hline Squamous cell carcinoma (SCC) & $42(42 \%)$ & $0(0 \%)$ & $18(31 \%)$ & $24(86 \%)$ & \\
\hline Not otherwise specified (NOS) & $8(8 \%)$ & $0(0 \%)$ & $4(7 \%)$ & $4(14 \%)$ & \\
\hline Unknown & $2(2 \%)$ & $1(8 \%)$ & $1(2 \%)$ & $0(0 \%)$ & \\
\hline 1 & $10(10 \%)$ & $1(8 \%)$ & $6(10 \%)$ & $3(11 \%)$ & \\
\hline 2 & $17(17 \%)$ & $2(17 \%)$ & $10(17 \%)$ & $5(18 \%)$ & \\
\hline 3 & $30(30 \%)$ & $3(25 \%)$ & $20(34 \%)$ & $7(25 \%)$ & \\
\hline 4 & $40(40 \%)$ & $5(42 \%)$ & $22(37 \%)$ & $13(46 \%)$ & \\
\hline $\mathrm{N}$-stage & & & & & 0.35 (N0-2 vs. N3) \\
\hline 0 & $10(10 \%)$ & $2(17 \%)$ & $5(8 \%)$ & $3(11 \%)$ & \\
\hline 1 & $9(9 \%)$ & $1(8 \%)$ & $6(10 \%)$ & $2(7 \%)$ & \\
\hline 2 & $36(36 \%)$ & $4(33 \%)$ & $21(36 \%)$ & $11(39 \%)$ & \\
\hline 3 & $44(44 \%)$ & $5(42 \%)$ & $27(46 \%)$ & $12(43 \%)$ & \\
\hline Tumor localisation & & & & & 0.611 \\
\hline Central & $40(40 \%)$ & $6(50 \%)$ & $19(32 \%)$ & 15 (54\%) & \\
\hline Pancoast & $7(7 \%)$ & $0(0 \%)$ & 7 (12\%) & $0(0 \%)$ & \\
\hline Lobular & 52 (53\%) & $6(50 \%)$ & 33 (56\%) & 13 (46\%) & \\
\hline
\end{tabular}


Table 2 Treatment characteristics

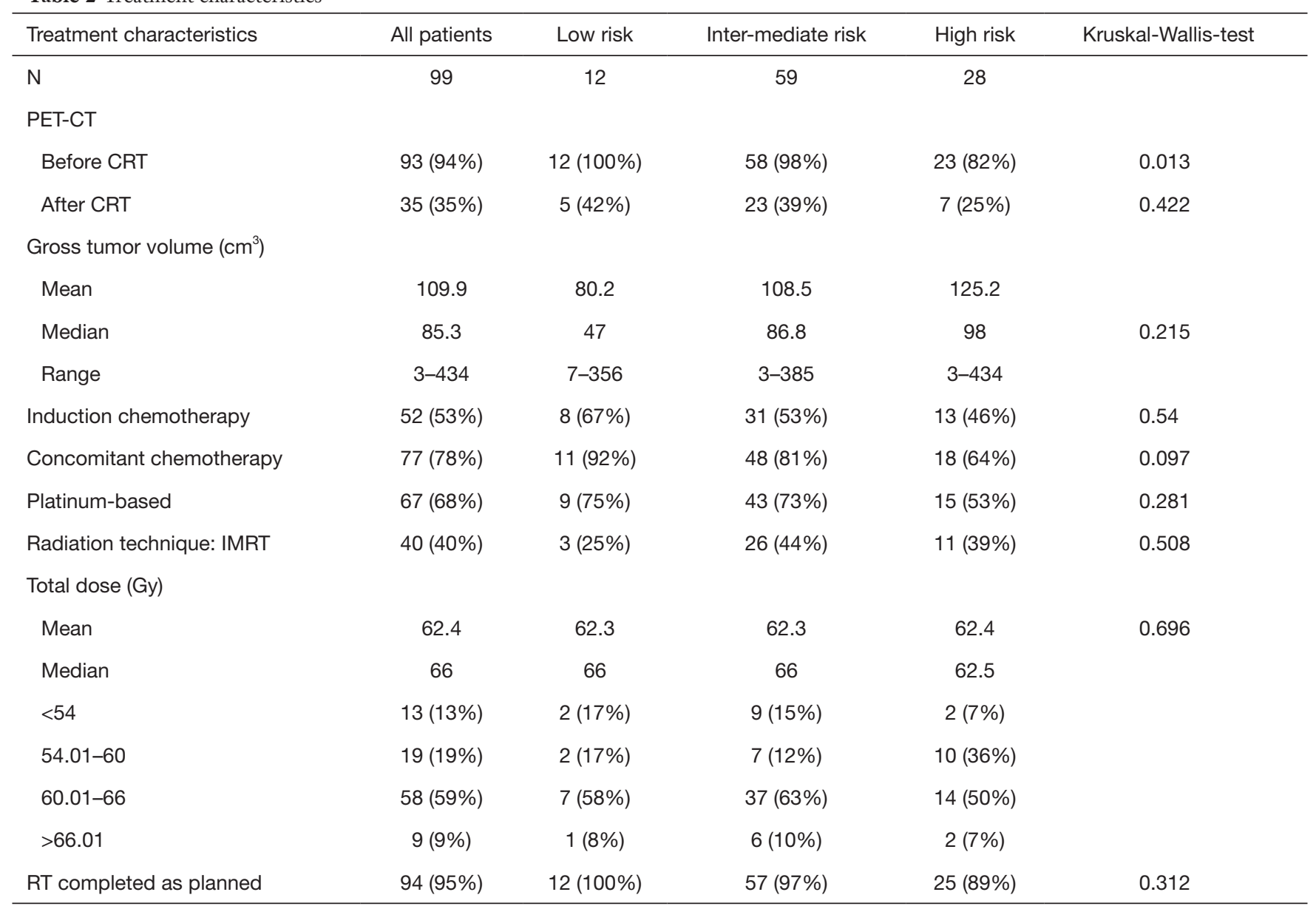

the whole course of radiotherapy. The therapy was stopped at a median of 58 Gy (range, 49-62 Gy) because of severe pneumonia (3 patients), myocardial infarction (1 patient) and Pulmonary embolism (1 patient).

In the first year after CRT, 29 patients (29\%) showed complete remission and 47 patients (47\%) partial remission in PET-CT or CT scans. During the observed period, $40 \%$ of patients received at least one course of salvage chemotherapy, immunotherapy or combined chemo-immunotherapy because of progressive disease. Seven percent of patients underwent planned surgery and $4 \%$ salvage surgery after completion of radiotherapy, $31 \%$ received salvage radiotherapy because of metastasis or progression.

\section{Toxicity, overall and event free survival}

Rates of radiation pneumonitis and esophagitis did not differ significantly in the risk subgroups. During treatment, 50 patients developed acute esophagitis; grade 3 disease was noted only in 4 patients (4\%). Radiation pneumonitis occurred in 25 patients after treatment; 4 patients (4\%) presented with grade 3 pneumonitis. No grade 4 and 5 nonhematological toxicity occurred (Table 3).

Median event-free survival (EFS) (time to local progression, distant metastasis or death) after CRT start was 8.7 months for all patients. Patients' age, gender and tobacco consumption had no impact on EFS with $\mathrm{P}$ values of $0.727,0.195$ and 0.150 , respectively. Patients with atelectasis had a median EFS of 4.3 months compared to 9.4 months for those without $(\mathrm{P}=0.028)$. An inferior median EFS was also observed for SCC and NOS versus patients with adenocarcinoma with 7.8 and 10.8 months, respectively $(\mathrm{P}=0.018)$ (panels A to E in Figure 1).

Median OS for all patients was 20.8 months [95\% confidence interval (CI): 15.3-26.3]. Patients aged 60 years or younger at initial diagnosis (26\%) showed a significantly improved OS with median OS of $26.4 v s .19 .3$ months in the patients aged $>60$ years, respectively $(\mathrm{P}=0.020)$. Female 
Table 3 Outcome, adverse events and survival

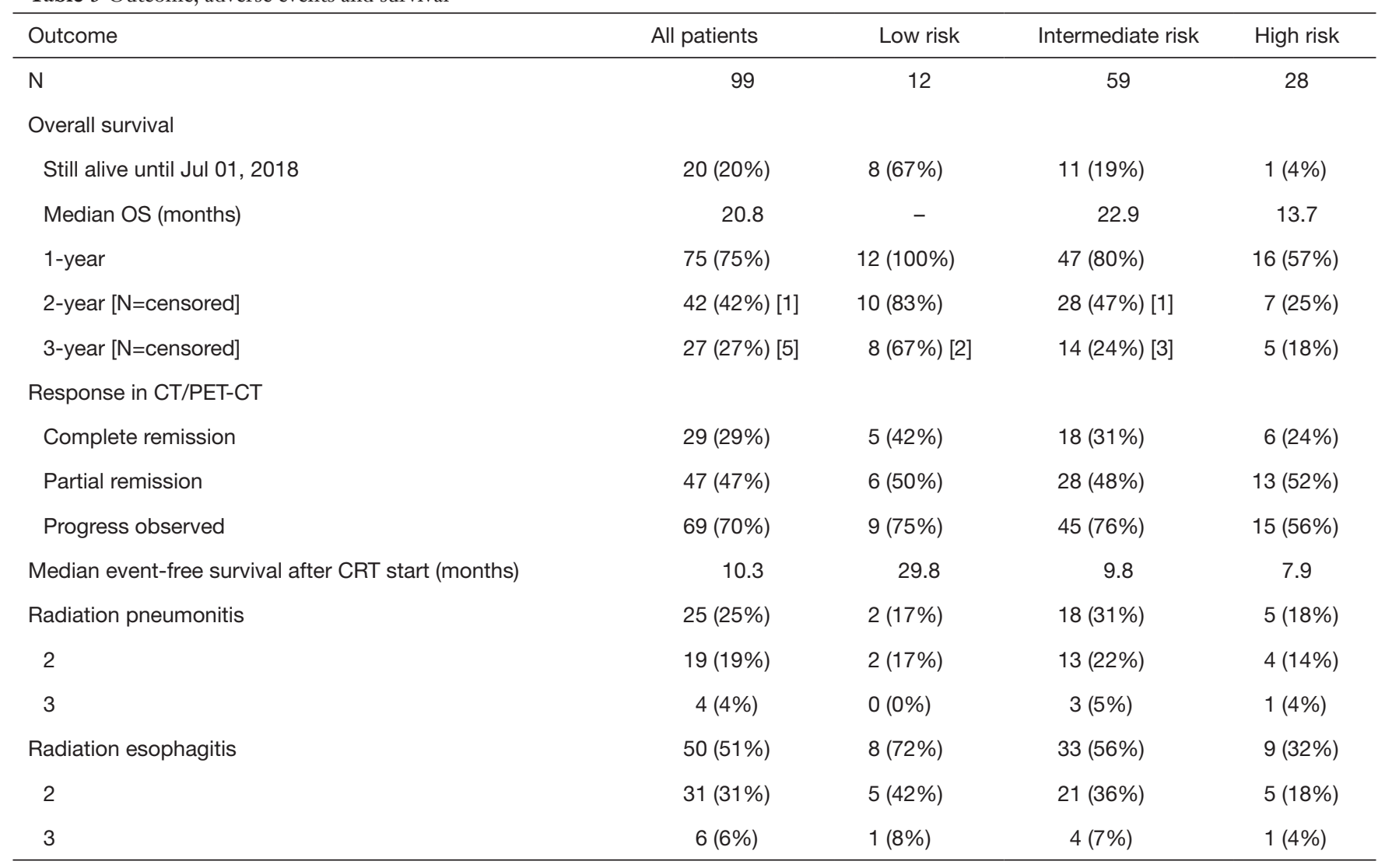

gender (37\%) also appeared to be a significant protective prognostic factor with a median OS of 31.4 months $v s$. 16.9 months for men $(\mathrm{P}=0.007)$. Patients who had smoked 20 pack years (PY) or more (82\%) displayed a significantly worse median OS of $19.1 \mathrm{vs.} 40.3$ months for those with less than $20 \mathrm{PY}(\mathrm{P}=0.015)$. Tumor related lung atelectasis before irradiation $(10 \%)$ was a significant negative predictor for median OS, with 8.8 vs. 22.0 months $(\mathrm{P}=0.004)$. Significant negative impact on median OS was also detected for SCC and NOS $(50 \%)$ vs. adenocarcinoma $(50 \%)$ with a median OS of 18.2 vs. 27.9 months respectively $(\mathrm{P}=0.011)$ (Figure $2 A, B, C, D, E)$.

Five factors significant for OS were included in a multivariate analysis: for patients older than 60 years the hazard ratio (HR) was 1.531 (95\% CI: 0.862-2.720; $\mathrm{P}=0.146$ ), for male patients the HR was 1.745 (95\% CI: 1.057-2.881; $\mathrm{p}=0.030$ ), for patients with 20 or more PY of tobacco consumption the HR was 1.841 (95\% CI: 0.931-3.640; $\mathrm{P}=0.079)$, for atelectasis before CRT HR was $2.359(95 \%$ CI: $1.180-4.717 ; \mathrm{P}=0.015)$ and for patients with SCC and NOS the HR was 1.326 (95\% CI: 0.826-2.128; $\mathrm{P}=0.243$ ).

\section{Single-center score}

All factors showing a significant negative impact on survival on univariate analysis were included in our heterogeneity score and weighted with one point each. Three subgroups were then defined: low risk (0-1 points) with 12 patients, intermediate risk (2-3 points) consisting of 59 patients and 28 patients with high risk (4-5 points).

The principal patient and tumor characteristics such as ECOG-PS, tumor stage and completeness of multimodal therapy did not differ significantly between risk subgroups, according to Kruskal-Wallis-testing (Table 2). We only found significantly more PET-CT based CRT-planning in our low and intermediate risk subgroup. Also, there was no difference in the rates of radiation pneumonitis and esophagitis between the subgroups.

EFS after the first day of CRT (including DM, LP or death) was 29.8 months (95\% CI: 23.8-62.3), 9.8 months (95\% CI: 11.1-20.1) and 7.9 months (95\% CI: 9.2-23.3) for the low, intermediate and high-risk subgroup respectively $(\mathrm{P}=0.036)$ (Figure 1F). 

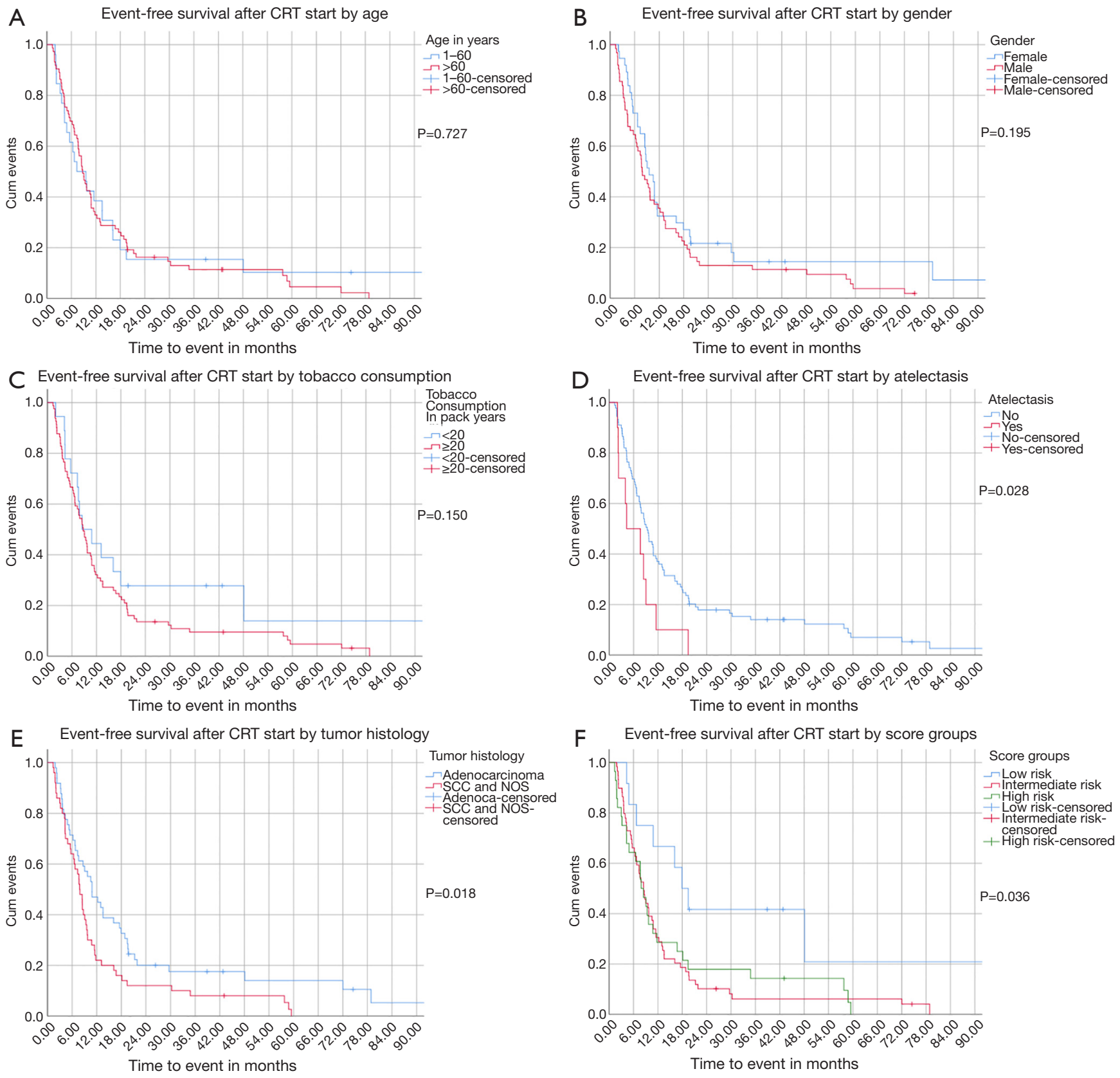

Figure 1 Event-free survival (EFS) by (A) age, (B) gender, (C) tobacco consumption, (D) atelectasis, (E) tumor histology, (F) score groups.

Median OS was not yet reached at the end of surveillance in our low risk subgroup, 1-year survival was 100\%, 2-year survival was $83 \%$ and 3 -year survival was $67 \%$. For the intermediate risk subgroup, median OS was 22.9 months (95\% CI: 16.3-29.5), 1-year survival was 95\%, 2-year survival was $80 \%$ and 3 -year survival was $24 \%$.

In the high-risk subgroup, median OS was 13.7 months, 1-year survival was $57 \%$, 2-year survival was $25 \%$ and 3 -year survival was $18 \%(\log$ rank: $\mathrm{P}<0.001)$ (Table 3; Figure $2 F)$.

Calculated with Cox regression the HR compared to all patients for the intermediate risk group was near the baseline (1.079). The HR for the low risk subgroup was 2.129 (95\% CI: $1.331-3.405 ; \mathrm{P}=0.002)$. In the low risk subgroup, the HR was 0.184 (95\% CI: 0.067-0.511; $\mathrm{P}=0.001)$. 

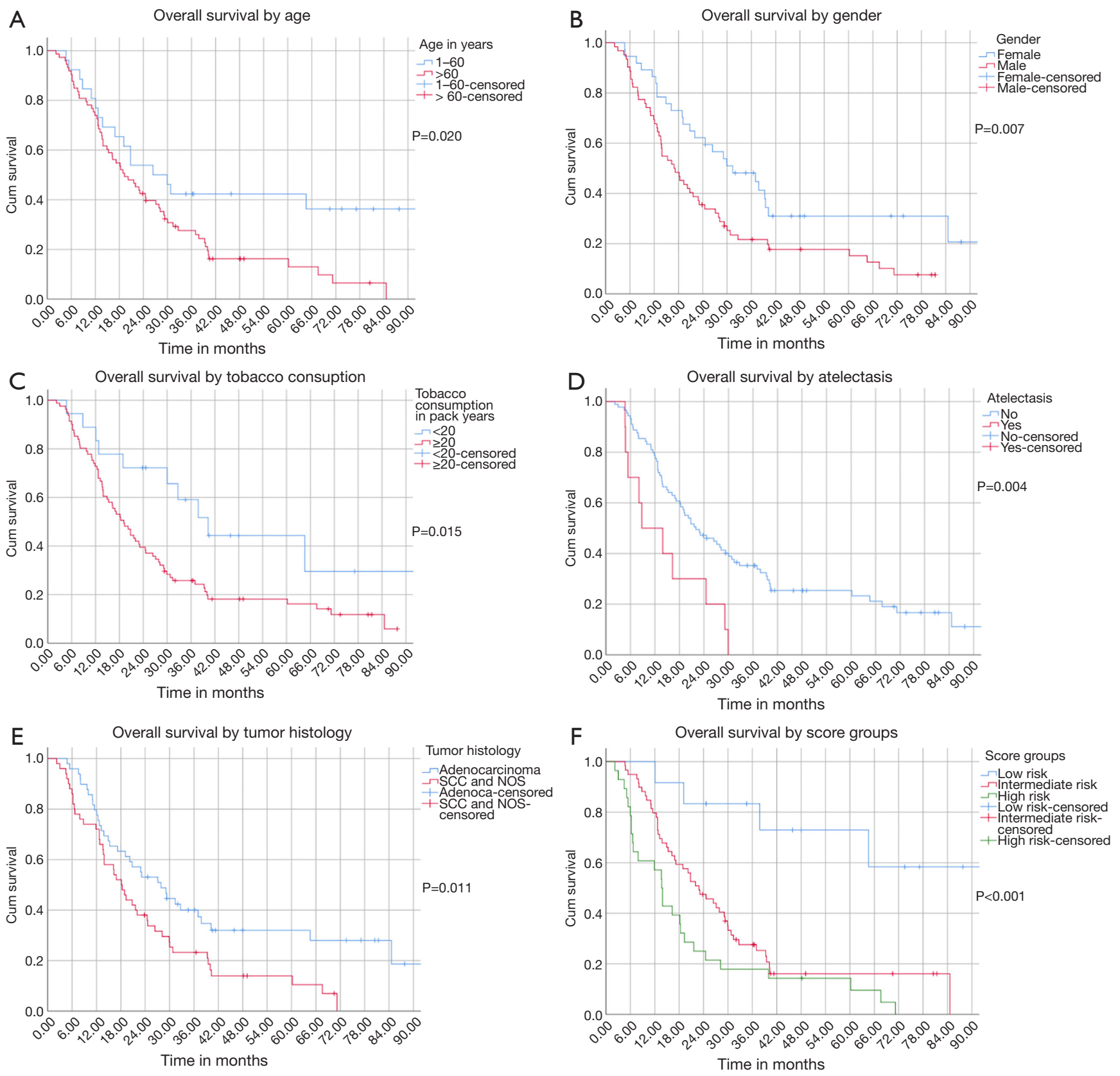

Figure 2 Overall survival (OS) by (A) age, (B) gender, (C) tobacco consumption, (D) atelectasis, (E) tumor histology, (F) score groups.

\section{Validation in own prospective cobort}

Median overall survival was not reached in the prospective validation cohort, median follow-up was 18 months (range: 5-30 months). Tumor related atelectasis was diagnosed in $11(24 \%)$ of patients. One-year survival was $45 \%$ for them compared to $96 \%$ for patients without atelectasis $(\mathrm{P}=0.003)$. The majority of $76 \%$ were male patients, 1 -year survival was $78 \%$ for male and $100 \%$ for female patients $(\mathrm{P}=0.064)$. Sixty-four percent of patients were older than 60 years at initial diagnosis with no difference in 1-year survival observed compared to those younger $(\mathrm{P}=0.999)$. The majority of $34(76 \%)$ patients had smoked more than 20 PY, 1-year survival was $83 \%$ vs. $89 \%$ for those with less than $20 \mathrm{PY}(\mathrm{P}=0.518)$. For SCC and NOS (19 patients; $64 \%$ ) vs. Adenocarcinoma (16 patients; $36 \%$ ) no significant 


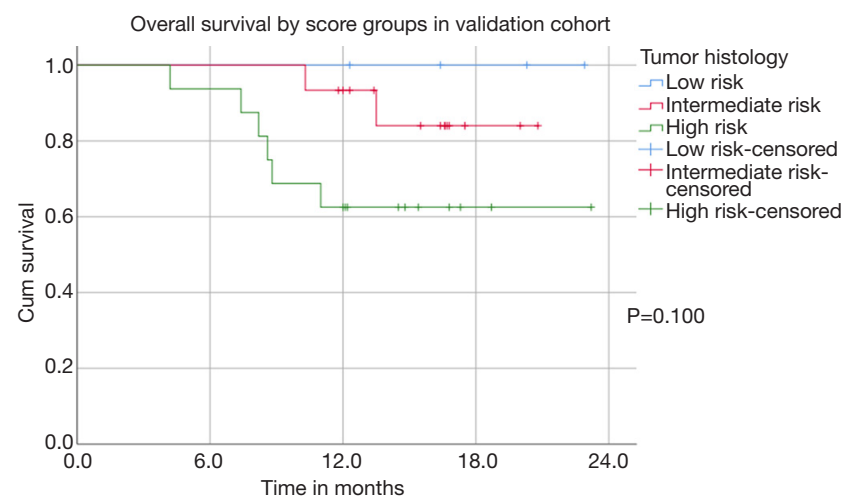

Figure 3 Overall survival in the validation cohort by score groups.

differences in 1-year survival could be detected wit $81 \% v s$. $92 \%$ respectively $(\mathrm{P}=0.598)$.

According to the score four $(11 \%)$ patients were sorted to the low risk, 15 patients (43\%) to the intermediate and $16(46 \%)$ patients to the high risk. One-year survival was $83 \%$ for all patients, $100 \%$ for the low risk, $94 \%$ for the intermediate risk and $70 \%$ for the high-risk subgroup $(\mathrm{P}=0.100)$. Median EFS after the first day of CRT was 12.6 months for all patients 15.1, 12.2 and 5.9 months for the low, intermediate and high-risk subgroups respectively ( $\mathrm{P}=0.257)$ (Figure 3).

\section{Discussion}

In inoperable stage III NSCLC, multimodal therapy will eventually transition from universal approach based on the motto "one size fits all" to the tailoring of treatment algorithm according to principal patient and tumor characteristics. Heterogeneity of stage III disease remains a challenge, has a direct impact on patient prognosis and is responsible for controversial results in prospective studies concerning treatment escalation. The scaling of heterogeneity is necessary to optimize development of multimodal approach regarding radiation dose prescription, consolidation with checkpoint inhibitors etc.

In this study, we report on a simple score for patients with good ECOG-PS and inoperable stage III NSCLC treated with CRT. The score is generated on basis of comprehensive analysis of patient- and tumor-related factors and to be easily applied. The score consists of five basic parameters that will be evaluated before starting multimodal therapy in any case. Therefore, it does not lead to a further delay before start of treatment or accrue costs if applied. We decided to use rounded cut-offs for age and PY, as well as one point for each parameter in the score, to keep it easy to calculate and usable in clinical routine.

Defined low, intermediate and high-risk subgroups demonstrated remarkable differences in event-free and overall survival even though there were no significant differences according to ECOG-PS, tumor stage and applied multimodal treatment. Patients with inoperable stage III NSCLC and a low-risk had an excellent prognosis with estimated 2- and 3-year survival rates of $83 \%$ and $67 \%$, respectively. In contrast, outcome in the high-risk patients was dismal despite adequate therapy with a median survival of 13.7 months and 2-/3-year survival rates of only $25 \%$ and $18 \%$. A prospective score validation with 1 year of followup and 45 patients did deliver a trend for differing survival between the risk subgroups. One-year survival ranged from $70 \%$ in the high to $100 \%$ in the low risk patients.

Importantly, patients included in the present analysis were treated at a high-volume tertiary cancer center. All diagnostic procedures including pathology and comprehensive imaging were performed at the same institution. There was a very high rate of initial ${ }^{18} \mathrm{~F}-\mathrm{FDG}$ PET/CT (94\%) in the analyzed cohort. In all cases, the decision to treat with CRT was confirmed by a multidisciplinary tumor-board.

The provided score has demonstrated how different patient prognosis in inoperable stage III NSCLC treated with multimodal approach could be. The event-free and overall survival in the low-risk subgroup was significantly better compared to historical data reported in the RTOG 0617 (21), GILT (22) and PROCLAIM (23) trials. Furthermore, survival of the low-risk subgroup was very similar to the data reported by Eberhardt et al. in the ESPATUE trial (24). However, multimodal approach in the ESPATUE was more intensive and included obligatory induction chemotherapy and accelerated radiation treatment protocol. Correspondingly, the rate of non-hematological severe toxicity was significantly higher compared to our present results.

Hallqvist et al. recently reported an excellent and similar with our low-risk subgroup survival in the conventional concurrent CRT arm (total dose of $68 \mathrm{~Gy}$ ) of a randomized phase II study in patients with good PS and inoperable stage III NSCLC (25). The study, however, revealed a highly negative effect of dose escalation (up to $84 \mathrm{~Gy}$ ) on patient survival in the experimental arm and was prematurely terminated.

Additionally, the achieved survival in our low-risk subgroup is in accordance with recently reported data from 
Dieleman et al. in stage III patients with ECOG 0-2 treated with concurrent daily low-dose cisplatin and moderate hypofractionated (2.75 Gy per fraction to total dose of $66 \mathrm{~Gy})$ thoracic irradiation (26). The authors stated that the treated population consisted of fit patients but exact data on ECOG-PS was missing. The reported rates of severe toxicity, first of all esophagitis were higher compared to our results. Iqbal et al. are following a similar approach with moderate hypofractionation concurrent CRT (55 Gy in 20 daily fractions concurrently with split-dose cisplatin vinorelbine chemotherapy) with promising results but slightly increased toxicity (27).

In contrast, conventional multimodal approach in patients with intermediate and, especially, high-risk needs further improvements. Whilst achieved survival for the intermediate risk cohort was principally in accordance with historical results, prognosis of the high-risk patients was very poor and more comparable with prognosis usually seen in metastatic disease. Patients with a high-risk score therefore represent the most challenging subgroup. Accumulation of patient- and treatment-related risk factors impede intensification of multimodal therapy.

In 1999, Movsas et al. has already reported on a potential negative survival impact of tumor histology and patient age based on the analysis of six prospective RTOG studies dedicated to treatment intensification (28). Analysis of 491 stage IIIA-B NSCLC patients treated with concurrent chemoradiation therapy published by Zhou et al. also revealed squamous cell histology as a strong local failure predictor (29).

This data suggests that patients with a high-risk score can benefit from a change of treatment paradigm, for example from conventionally fractionated thoracic irradiation to moderate hypofractionated concepts applied concurrently with chemotherapy.

Data reported from Dielemann et al. for patients treated concurrently with low-dose cisplatin and moderate hypofractionated thoracic irradiation seemed promising (26). The next step could also be the integration of checkpoint inhibition in the multimodal approach to moderate tumor immune surveillance, perhaps to further improve survival of intermediate or low risk patients. The pilot study of neo-adjuvant PD1-inhibition with nivolumab in resectable NSCLC was very promising with a major pathological response occurring in $45 \%$ of resected tumors after only two infusions of nivolumab (30). Initial survival data from the NICOLAS phase II trial investigating feasibility of concurrent chemoradioimmunotherapy with nivolumab in stage III NSCLC patients with ECOG 0-1 are also awaited and can potentially confirm a positive effect of concurrent and consolidation checkpoint inhibition on patient outcome (31). The recently published PACIFIC trial reported a historically best progression-free survival in patients with good PS and inoperable stage III NSCLC treated with CRT followed by consolidation treatment with Durvalumab (32).

It is important to consider the limitations of the present study. The score was based on comprehensive analysis of the follow-up data of 99 consecutive patients with good performance status, who completed initial diagnostic and staging procedures as well as multimodal therapy at a single high-volume university medical center. Hence, the next step will be validation of this heterogeneity score in external independent cohorts.

In summary, we present a simple score for inoperable stage III NSCLC patients with good PS treated with multimodal therapy. This score has identified remarkable overall survival differences after completion of CRT and suggested a need for further tailoring of the multimodal approach. The score has revealed an excellent long-term outcome of the low-risk patients but confirmed that utmost efforts are required to improve OS of patients in the highrisk subgroup.

\section{Conclusions}

In the present study we developed a simple score for inoperable stage III NSCLC patients with good performance status receiving CRT. The scaling of heterogeneity in stage III is necessary to further optimize a multimodal treatment.

Defined low, intermediate and high-risk score subgroups demonstrated remarkable differences in event-free and overall survival even though there were no significant differences according to ECOG-PS, tumor stage and applied treatment.

The score may aid physicians to infer patient clinical outcomes and optimize everyday decision-making.

\section{Acknowledgments}

None.

\section{Footnote}

Conflicts of Interest: The authors have no conflicts of interest to declare. 
Ethical Statement: The authors are accountable for all aspects of the work in ensuring that questions related to the accuracy or integrity of any part of the work are appropriately investigated and resolved. All patients gave express written informed consent. This retrospective analysis is in compliance with the principles of the Declaration of Helsinki and its subsequent amendments. The study was approved by the Ethics Committee of the Ludwig Maximilian University of Munich (No. 17-230). The datasets used and analysed during the current study are available from the corresponding author on reasonable request.

\section{References}

1. Ferlay J, Soerjomataram I, Dikshit R, et al. Cancer incidence and mortality worldwide: sources, methods and major patterns in GLOBOCAN 2012. Int J Cancer 2015;136:E359-86.

2. Midha A, Dearden S, McCormack R. EGFR mutation incidence in non-small-cell lung cancer of adenocarcinoma histology: a systematic review and global map by ethnicity (mutMapII). Am J Cancer Res 2015;5:2892-911.

3. W. SB, P. WC. World Cancer Report 2014. Geneva: World Health Organization; 2014. Available online: http:// gbv.eblib.com/patron/FullRecord.aspx?p=1635715

4. Robert Koch-Institut. Krebs in Deutschland für 2013/2014.

5. Westover KD, Loo BW, Gerber DE, et al. Precision Hypofractionated Radiation Therapy in Poor Performing Patients With Non-Small Cell Lung Cancer: Phase 1 Dose Escalation Trial. Int J Radiat Oncol Biol Phys 2015;93:72-81.

6. Swanick CW, Lin SH, Sutton J, et al. Use of Simultaneous Radiation Boost Achieves High Control Rates in Patients With Non-Small-Cell Lung Cancer Who Are Not Candidates for Surgery or Conventional Chemoradiation. Clin Lung Cancer 2015;16:156-63.

7. Skrzypski M, Jassem J. Consolidation systemic treatment after radiochemotherapy for unresectable stage III nonsmall cell lung cancer. Cancer Treat Rev 2018; 66:114-21.

8. Pollom EL, Qian Y, Durkee BY, et al. Hypofractionated Intensity-Modulated Radiotherapy for Patients With Non-Small-Cell Lung Cancer. Clin Lung Cancer 2016;17:588-94.

9. Manapov F, Roengvoraphoj O, Li M, et al. Moderate hypofractionated image-guided thoracic radiotherapy for locally advanced node-positive non-small cell lung cancer patients with very limited lung function: a case report. Radiat Oncol J 2017;35:180-4.

10. Maguire J, Khan I, McMenemin R, et al. SOCCAR: A randomised phase II trial comparing sequential versus concurrent chemotherapy and radical hypofractionated radiotherapy in patients with inoperable stage III NonSmall Cell Lung Cancer and good performance status. Eur J Cancer 2014;50:2939-49.

11. Brahmer JR, Govindan R, Anders RA, et al. The Society for Immunotherapy of Cancer consensus statement on immunotherapy for the treatment of non-small cell lung cancer (NSCLC). J Immunother Cancer 2018;6:75.

12. Feliciano JL, Le-Rademacher JG, Gajra A, et al. Do older patients with non-small cell lung cancer also benefit from first-line platinum-based doublet chemotherapy? Observations from a pooled analysis of 730 prospectivelytreated patients (Alliance Study A151622). J Geriatr Oncol 2018;9:501-6.

13. Mahar AL, Compton C, McShane LM, et al. Refining Prognosis in Lung Cancer. J Thorac Oncol 2015;10:1576-89.

14. Brundage MD, Davies D, Mackillop WJ. Prognostic factors in non-small cell lung cancer: a decade of progress. Chest 2002;122:1037-57.

15. Chermiti Ben Abdallah F, Ben Ali G, Sadok Boudaya $\mathrm{M}$ et al., Traitement et pronostic du cancer bronchique non à petites cellules au stade avancé. Rev Mal Respir 2014;31:214-20.

16. Dutkowska AE, Antczak A. Comorbidities in lung cancer. Pneumonol Alergol Pol 2016;84:186-92.

17. Forrest LM, McMillan DC, McArdle CS, et al. Evaluation of cumulative prognostic scores based on the systemic inflammatory response in patients with inoperable nonsmall-cell lung cancer. Br J Cancer 2003;89:1028-30.

18. Alexander M, Wolfe R, Ball D, et al. Lung cancer prognostic index: a risk score to predict overall survival after the diagnosis of non-small-cell lung cancer. Br J Cancer 2017;117:744-51.

19. Gagnon B, Agulnik JS, Gioulbasanis I, et al. Montreal prognostic score: estimating survival of patients with non-small cell lung cancer using clinical biomarkers. $\mathrm{Br} \mathrm{J}$ Cancer 2013;109:2066-71.

20. Rades D, Käsmann L, Schild SE, et al. A Survival Score for Patients Receiving Palliative Irradiation for Locally Advanced Lung Cancer. Clin Lung Cancer 2016;17:558-62.

21. Bradley JD, Paulus R, Komaki R, et al. Standard-dose versus high-dose conformal radiotherapy with concurrent and consolidation carboplatin plus paclitaxel with or 
without cetuximab for patients with stage IIIA or IIIB non-small-cell lung cancer (RTOG 0617): a randomised, two-by-two factorial phase 3 study. Lancet Oncol 2015;16:187-99.

22. Flentje M, Huber RM, Engel-Riedel W, et al. GILT-A randomised phase III study of oral vinorelbine and cisplatin with concomitant radiotherapy followed by either consolidation therapy with oral vinorelbine and cisplatin or best supportive care alone in stage III non-small cell lung cancer. Strahlenther Onkol 2016;192:216-22.

23. Senan S, Brade A, Wang LH, et al. PROCLAIM: Randomized Phase III Trial of Pemetrexed-Cisplatin or Etoposide-Cisplatin Plus Thoracic Radiation Therapy Followed by Consolidation Chemotherapy in Locally Advanced Nonsquamous Non-Small-Cell Lung Cancer. J Clin Oncol 2016;34:953-62.

24. Eberhardt WEE, Pöttgen C, Gauler TC, et al. Phase III Study of Surgery Versus Definitive Concurrent Chemoradiotherapy Boost in Patients With Resectable Stage IIIA(N2) and Selected IIIB Non-Small-Cell Lung Cancer After Induction Chemotherapy and Concurrent Chemoradiotherapy (ESPATUE). J Clin Oncol 2015;33:4194-201.

25. Hallqvist A, Bergström S, Björkestrand H, et al. Dose escalation to 84 Gy with concurrent chemotherapy in stage III NSCLC appears excessively toxic: Results from a prematurely terminated randomized phase II trial. Lung Cancer 2018;122:180-6.

26. Dieleman EMT, Uitterhoeve ALJ, van Hoek MW, et al. Concurrent daily Cisplatin and high dose radiotherapy

Cite this article as: Taugner J, Käsmann L, Eze C, Dantes M, Roengvoraphoj O, Gennen K, Karin M, Petruknov O, Tufman A, Belka C, Manapov F. Survival score to characterize prognosis in inoperable stage III NSCLC after chemoradiotherapy. Transl Lung Cancer Res 2019;8(5):593-604. doi: 10.21037/ tlcr.2019.09.19 in patients with stage III non-small cell lung cancer. Int J Radiat Oncol Biol Phys 2018;102:543-51.

27. Iqbal MS, Vashisht G, McMenemin R, et al. Hypofractionated Concomitant Chemoradiation in Inoperable Locally Advanced Non-small Cell Lung Cancer: A Report on 100 Patients and a Systematic Review. Clin Oncol (R Coll Radiol) 2019;31:e1-10.

28. Movsas B, Scott C, Sause W, et al. The benefit of treatment intensification is age and histology-dependent in patients with locally advanced non-small cell lung cancer (NSCLC): a quality-adjusted survival analysis of radiation therapy oncology group (RTOG) chemoradiation studies. Int J Radiat Oncol Biol Phys 1999;45:1143-9.

29. Zhou R, Xu T, Nguyen QN, et al. Radiation Dose, Local Disease Progression, and Overall Survival in Patients With Inoperable Non-Small Cell Lung Cancer After Concurrent Chemoradiation Therapy. Int J Radiat Oncol Biol Phys 2018;100:452-61.

30. Forde PM, Chaft JE, Smith KN, et al. Neoadjuvant PD-1 Blockade in Resectable Lung Cancer. N Engl J Med 2018;378:1976-86.

31. NIvolumab COmbination With Standard First-line Chemotherapy and Radiotherapy in Locally Advanced Stage IIIA/B Non-Small Cell Lung Carcinoma. [cited 2018 Sep 1]. Available online: https://clinicaltrials.gov/ct2/ show/NCT02434081

32. Uemura T, Hida T. Durvalumab showed long and durable effects after chemoradiotherapy in stage III non-small cell lung cancer: results of the PACIFIC study. J Thorac Dis 2018;10:S1108-12. 